conversation, checking the recipient's welfare, and how to open and close the conversations.

Conclusion The unique feedback from the simulated relative's perspective has enabled us to deliver a learning package that helps to prepare learners to deliver unwelcome news over the telephone in time critical situations. Further work will look at the clinical impact of this project.

\section{INTRODUCTION OF THE ROLE OF ASSISTANT PRACTITIONER IN A PALLIATIVE CARE SETTING}

Esther Webb, Tracey Taylor, Lorna Richardson, Sarah Stanley. Marie Curie

10.1136/spcare-2021-PCC.69

Introduction The role of Assistant Practitioner (AP) was first formally defined in 2002 and is widely considered a pioneering role able to work semi-autonomously across various health and social care settings. Although not registered to a formal body, an AP's accountability comes through locally agreed and defined protocols. Health Care Assistants (HCA) can progress to AP by successfully completing a 2-year Foundation Degree in Health and Social Care. In 2015 Marie Curie Hospice Liverpool introduced the role of AP to work across all patientfacing areas and two individuals were identified to attend university one day a week while spending 4 days per week in practice developing new skills.

Methods Due to the role being new to the charity, there was opportunity for the AP to co-create the job specification and identify, with the help of the wider multi-disciplinary team (MDT), tasks which would be beneficial to the team for the AP to carry out. The AP would continue to carry out many of the tasks usually delegated to the HCA, while also developing new skills to support the registered nurse.

Results Both APs successfully completed the Foundation Degree and continued to develop the role and their skills. New opportunities to develop the role are continuously sought although there have been some barriers to development due to not being registered to a formal body; mainly around medication checking and administration. Nevertheless, venepuncture, assessing new patients, handover of patients to the MDT and assisting in the application of topical medications have become key skills within the role of AP.

Conclusion Although the introduction and implementation of the role has been somewhat successful, there are areas of the journey of the development of the role that have been identified that could have been improved on and role distinction is still unclear to a certain degree.

\section{A VIRTUAL MULTIDISCIPLINARY JOURNAL CLUB}

Sarah Stanley, Helen Bonwick, Laura Chapman, Amara Nwosu. Marie Curie Hospice Liverpool, University of Lancaster, Royal Liverpool Hospitals NHS Foundation Trust

\subsection{6/spcare-2021-PCC.70}

Background Research is essential in enabling practitioners to deliver evidence-based treatment and care. ${ }^{1}$ A journal club has been identified as a way to encourage a research culture by engaging staff to build evidence of best practice. ${ }^{2}$ Since January 2019 Marie Curie Hospice Liverpool have developed a unique multi-disciplinary approach to journal club, which has recently been impacted by the COVID19 pandemic. We recognised the importance of re-instating these sessions in order to continue learning and development of a research culture in our hospice.

Methods Our journal club is held fortnightly and is co-ordinated by the research nurse and speciality doctor with support from our research lead. Following the height of the COVID19 pandemic, meetings resumed and were encouraged to utilise Microsoft teams in order to maintain social distancing in meeting rooms. This provided us with a unique opportunity to expand our journal club outside of the hospice and invite healthcare professionals from external organisations (including acute hospital trusts and other local hospices) to join these meetings.

Results To date we have held a total of 22 meetings. Healthcare professionals attending the meetings have generated 109 research questions relevant to palliative care. Feedback from external organisations has been positive, and these healthcare professionals report being grateful for the opportunity to attend a journal club. We are currently working on developing a structure for a quality improvement project which will allow members of the MDT to come together and answer one of our generated questions.

Conclusions Our MDT journal club model has proved to be successful, and with the support of technology has allowed us to share our learning outside of the hospice. Attendance and engagement across disciplines has made our staff research aware, creating a culture of enquiry and an eagerness to provide evidence-based care.

\section{REFERENCES}

1. Preston NJ, Payne SA and Todd C. Conducting research in palliative care patients: burden or an opportunity? International journal of Palliative Nursing 2009;15 (11):524-525

2. Payne $\mathrm{S}$, Preston N, Turner M, Rolls L. Research in palliative care - can hospices afford to not be involved? International Observatory on End of Life Care 2013.

\section{AN AUDIT INVESTIGATING THE ROLE OF NASAL HIGH FLOW OXYGEN THERAPY IN ONCOLOGY PATIENTS - IS IT DELAYING APPROPRIATE PALLIATION?}

Seema Green, Adriana Pereira. Gloucester Royal Hospital, Cheltenham General Hospital

\subsection{6/spcare-2021-PCC.71}

Introduction High-flow nasal oxygen (HFNO) is increasingly used as part of ward-based treatment, especially for oncology patients with respiratory failure that is either directly due to the underlying malignancy or secondary to anti-cancer drugs or radiotherapy-induced pneumonitis. Patient selection is paramount to its success, as unsuitable selection can result in delays in appropriate palliation, leading to unrealistic expectations for relatives.

Methods We performed a retrospective audit of 40 patients across both Gloucester Royal and Cheltenham General Hospitals, who received HFNO on the respiratory wards over JulyDecember 2019.

Results The average age for patients receiving HFNO was 74 years with mortality rates being the highest at $80 \%$ in the $80-89$ years age group. Interestingly, $81 \%$ of patients with HFNO as their ceiling of treatment died and only $10 \%$ of patients deemed for full escalation of treatment died. The majority of referrals were from medical specialities, closely followed by oncology. The oncology patients did show the highest mortality rates. The majority of patients referred for 